\title{
Avaliação das atividades antimicrobiana e citotóxica de frações ricas em alcaloides obtidas das partes aéreas de Mitracarpus frigidus (Rubiaceae)
}

\author{
Evaluation of the antimicrobial and cytotoxic activities of alkaloid-rich fractions obtained from
}

Mitracarpus frigidus aerial parts (Rubiaceae)

Evaluación de las actividades antimicrobianas y citotóxicas de fracciones ricas en alcaloides obtenidas de las partes aéreas de Mitracarpus frigidus (Rubiaceae)

Recebido: 17/08/2021 | Revisado: 22/08/2021 | Aceito: 24/08/2021 | Publicado: 25/08/2021

\author{
Thalita de Freitas Souza \\ ORCID: https://orcid.org/0000-0002-7316-2233 \\ Universidade Federal de Juiz de Fora, Brasil \\ E-mail: thalitadf.souza@gmail.com \\ Lara Melo Campos \\ ORCID: https://orcid.org/0000-0003-3039-0559 \\ Universidade Federal de Juiz de Fora, Brasil \\ E-mail: laramelo95@yahoo.com.br \\ Ari Sérgio de Oliveira Lemos \\ ORCID: https://orcid.org/0000-0002-2980-7647 \\ Universidade Federal de Juiz de Fora, Brasil \\ E-mail: arisergiolemos@ hotmail.com \\ Irley Olívia Mendonça Diniz \\ ORCID: https://orcid.org/0000-0003-4449-624X \\ Universidade Federal de Juiz de Fora, Brasil \\ E-mail: olivia.diniz95@gmail.com \\ Priscila de Lima Paula \\ ORCID: https://orcid.org/0000-0002-6848-6604 \\ Universidade Federal de Juiz de Fora, Brasil \\ E-mail: plppriscila23@gmail.com \\ Marcelo Gonzaga de Freitas Araújo \\ ORCID: https://orcid.org/0000-0002-6320-3185 \\ Universidade Federal de São João Del Rei, Brasil \\ E-mail:mgfaraujo@ufsj.edu.br \\ Luciana Moreira Chedier \\ ORCID: https://orcid.org/0000-0001-8212-8056 \\ Universidade Federal de Juiz de Fora, Brasil \\ E-mail:1mchedier@hotmail.com \\ Rodrigo Luiz Fabri \\ ORCID: https://orcid.org/0000-0002-0167-2277 \\ Universidade Federal de Juiz de Fora, Brasil \\ E-mail: rodrigo.fabri@ufjf.edu.br
}

\begin{abstract}
Resumo
O presente estudo teve como objetivo extrair e avaliar o potencial antimicrobiano e citotóxico de frações ricas em alcaloides obtidas a partir das partes aéreas de Mitracarpus frigidus. O isolamento de alcaloides foi realizado por meio de extração ácido-base e cromatografia em coluna com Sephadex LH-20 como fase estacionária. A atividade antimicrobiana foi avaliada pelo método de microdiluição em caldo para determinação da concentração inibitório mínima (CIM) e também por parâmetros quantitativos antimicrobianos (atividade total, percentual de atividade e índice de susceptibilidade microbiano) contra nove linhagens de microrganismos: Staphylococcus aureus, Pseudomonas aeruginosa, Salmonella typhimurium, Shigella sonnei, Klebsiella pneumoniae, Escherichia coli, Bacillus cereus, Candida albicans e Cryptococcus neoformans. A citotoxicidade foi testada frente a Artemia salina e duas linhagens de células leucêmicas, HL60 e Jurkart. A fração mais rica em alcaloides apresentou atividade expressiva para S. aureus, S. typhimurium, B. cereus, P. aeruginosa, S. sonnei e C. neoforman com CIM abaixo de $0,100 \mathrm{mg} / \mathrm{mL}$. As amostras não apresentaram efeito citotóxico frente a A. salina e em relação as linhagens de células tumorais, somente as frações mais ricas em alcaloides apresentaram uma moderada atividade com cerca 50 e $35 \%$ de diminuição da viabilidade celular para HL60 e Jurkart, respectivamente. Os resultados apresentados abrem perspectivas para o futuro isolamento, purificação e identificação de substâncias bioativas de M. frigidus que possam ser utilizadas, principalmente no tratamento de infecções microbianas.
\end{abstract}

Palavras-chave: Rubiaceae; Mitracarpus frigidus; Alcaloides; Atividade antimicrobiana; Citotoxicidade. 


\begin{abstract}
The present study aimed to extract and evaluate the antimicrobial and cytotoxic potential of alkaloid-rich fractions obtained from Mitracarpus frigidus aerial parts. The isolation of alkaloids was performed by acid-base extraction and column chromatography with Sephadex LH-20 as stationary phase. The antimicrobial activity was evaluated by the broth microdilution method to determine the minimum inhibitory concentration (MIC) and also by antimicrobial quantitative parameters (total activity, percentage of activity and microbial susceptibility index) against nine strains of microorganisms: Staphylococcus aureus, Pseudomonas aeruginosa, Salmonella typhimurium, Shigella sonnei, Klebsiella pneumoniae, Escherichia coli, Bacillus cereus, Candida albicans and Cryptococcus neoformans. Cytotoxicity was tested against Artemia salina and two leukemic cell lines, HL60 and Jurkart. The fraction richest in alkaloid showed expressive activity for S. aureus, S. typhimurium, B. cereus, P. aeruginosa, S. sonnei and C. neoformans with MIC below $0.100 \mathrm{mg} / \mathrm{mL}$. The samples did not show cytotoxic effect against A. salina and, in relation to the tumor cell lines, only the fractions rich in alkaloids showed moderate activity with about 50 and $35 \%$ decrease in cell viability for HL60 and Jurkart, respectively. The presented results open perspectives for the future isolation, purification and identification of bioactive substances from M. frigidus that can be used, mainly in the treatment of microbial infections.
\end{abstract}

Keywords: Rubiaceae; Mitracarpus frigidus; Alkaloids; Antimicrobial activity; Cytotoxicity.

\title{
Resumen
}

El presente estudio tenía como objetivo extraer y evaluar el potencial antimicrobiano y citotóxico de las fracciones ricas en alcaloides obtenidas de las partes aéreas de Mitracarpus frigidus. El aislamiento de los alcaloides se realizó mediante extracción ácido-base y cromatografía en columna con Sephadex LH-20 como fase estacionaria. La actividad antimicrobiana se evaluó por el método de microdilución en caldo para determinar la concentración mínima inhibitoria (CIM) y también por parámetros cuantitativos antimicrobianos (actividad total, porcentaje de actividad e índice de susceptibilidad microbiana) contra nueve cepas de microorganismos: Staphylococcus aureus, Pseudomonas aeruginosa, Salmonella typhimurium, Shigella sonnei, Klebsiella pneumoniae, Escherichia coli, Bacillus cereus, Candida albicans y Cryptococcus neoformans. La citotoxicidad se probó contra Artemia salina y dos líneas celulares leucémicas, HL60 y Jurkart. La fracción más rica en alcaloides mostró una actividad expresiva para S. aureus, S. typhimurium, B. cereus, P. aeruginosa, S. sonnei y C. neoforman con CMI inferiores a 0,100 mg/mL. Las muestras no mostraron efecto citotóxico contra A. salina y en relación con las líneas celulares tumorales, sólo las fracciones más ricas en alcaloides mostraron una actividad moderada con cerca del 50 y 35\% de disminución de la viabilidad celular para HL60 y Jurkart, respectivamente. Los resultados presentados abren perspectivas para el futuro aislamiento, purificación e identificación de sustancias bioactivas de M. frigidus que puedan ser utilizadas, principalmente, en el tratamiento de infecciones microbianas.

Palabras clave: Rubiaceae; Mitracarpus frigidus; Alcaloides; Actividad antimicrobiana; Citotoxicidad.

\section{Introdução}

Os alcaloides são substâncias nitrogenadas farmacologicamente ativas e são encontrados principalmente nas angiospermas. Na sua grande maioria, possuem caráter alcalino, com exceções tais como colchicina, piperina, oximas e alguns sais quaternários como o cloridrato de laurifolina (Simões et al., 2011).

De acordo com Cordell et al. (2001), a família Rubiaceae possui cerca de 677 alcaloides já isolados e destes, mais da metade, 391, são alcaloides indólicos e benzilisoquinolínicos. Os alcaloides da família Rubiaceae possuem grande importância farmacológica, como por exemplo: a cafeína, isolada de Coffea arabica L. (estimulante do Sistema Nervoso Central); a emetina, isolada de Cephelis ipecacuanha Richaud. (amebecida e emético); a quinina e quinidina, isoladas de Cinchona ledgeriana Moens. ex Trimen (antimalárico e antiarrítmico, respectivamente) (Martins \& Nunez, 2015).

As espécies de Mitracarpus são caracterizadas por serem ricas em alcaloides. Okunade et al. (1999) isolaram um alcaloide com atividade antimicrobiana a partir do extrato etanólico das partes aéreas de M. scaber, azaantraquinona (benzilisoquinolina-5,10-diona). Outros trabalhos confirmaram a atividade antimicrobiana deste alcaloide e também descreveram ser um promissor composto como agente tripanomicida (Nok, 2002; Gbaguidi et al., 2005). Não há relatos de extração e isolamento de alcaloides em M. frigidus (Willd. ex Reem Schult.) K. Schum., uma planta encontrada em toda a América do Sul, principalmente no Brasil (Pereira et al., 2006) e já possui relatos de atividade antimicrobiana, leishmanicida e antioxidante (Fabri et al, 2009). Diante do ineditismo do estudo de alcaloides para M. frigidus, o objetivo desse trabalho foi a extração e isolamento de frações ricas em alcaloides obtidos de extratos orgânicos das partes aéreas dessa espécie, além da 
avaliação das atividades antimicrobiana e citotóxica.

\section{Metodologia}

\subsection{Material vegetal}

As partes aéreas de M. frigidus foram coletadas em Juiz de Fora, Minas Gerais, Brasil, em maio de 2009. A exsicata foi depositada no Herbário Leopoldo Krieger (CESJ 46076) da Universidade Federal de Juiz de Fora (SISGEN/ Brasil A032F41).

\subsection{Extração de alcaloides}

A extração de alcaloides foi realizada segundo protocolo descrito por Matos (1997), com algumas modificações. As partes aéreas de M. frigidus foram secas, pesadas (430g) e extraídas com hexano. Em seguida, o resíduo botânico foi seco em capela de exaustão e extraído exaustivamente com metanol por sete dias à temperatura ambiente. O extrato metanólico foi concentrado à pressão reduzida utilizando evaporador rotatório $(78 \mathrm{~g})$ e solubilizado em solução de ácido tartárico $2 \%$ (400 $\mathrm{mL}, \mathrm{pH}$ 3-4). Após extração com acetato de etila (4 x $200 \mathrm{~mL}$ ), a fase aquosa foi tratada com $\mathrm{Na}_{2} \mathrm{CO}_{3} 10 \%$ (pH 8-10) e extraída novamente com acetato de etila $(4$ x $200 \mathrm{~mL})$. Todas as etapas foram monitoradas pelo reativo de Dragendorff.

Da fase aquosa precipitou-se um material de aspecto escuro e pastoso, rico em alcaloides, que foi retirado e solubilizado em metanol (MFAM, 1,3 g) e submetido ao fracionamento em coluna cromatográfica de Sephadex LH-20 (Merck) $(80 \times 3 \mathrm{~cm}$ ), usando como fase móvel metanol. As frações obtidas foram agrupadas de acordo com o perfil cromatográfico em dez frações (MFAM1 a MFAM10) (fase móvel: MeOH:ácido acético (8:2); revelação: reativo de Dragendorff). As frações foram concentradas à pressão reduzida, utilizando evaporador rotatório, pesadas e mantidas sob refrigeração até a realização dos testes biológicos.

No extrato hexânico (15 g), também se verificou a presença de alcaloides (reação de Dragendorff positivo). Dessa forma, esse extrato também foi submetido ao procedimento de extração de alcaloides. Ao alcalinizar o extrato hexânico, um precipitado branco (MFAH, $50 \mathrm{mg}$ ) foi formado e, em seguida, separado do sobrenadante. Esse precipitado foi submetido à cromatografia de camada delgada - CCD (fase móvel: $\mathrm{MeOH}$ ácido acético (8:2); revelação: reativo de Dragendorff).

\subsection{Atividade Antimicrobiana}

\subsubsection{Microrganismos testados}

Os microrganismos testados foram Staphylococcus aureus (ATCC® 6538), Pseudomonas aeruginosa (ATCC®C 15442), Salmonella enterica sorovar typhimurium (ATCC® 13311), Shigella sonnei (ATCC® 11060), Klebsiella pneumoniae (ATCC® 13866), Escherichia coli (ATCC® 10536), Bacillus cereus (ATCC® 11778), Candida albicans (ATCC® 18804) e Cryptococcus neoformans (ATCC® 32608).

\subsubsection{Determinação da concentração inibitória mínima (CIM)}

O ensaio de susceptibilidade em microdiluição em caldo foi realizado usando o método descrito pela CLSI, 2010 para bactérias e CLSI, 2017 para fungos. As linhagens de bactérias foram cultivadas overnight a $35^{\circ} \mathrm{C}$ por $24 \mathrm{~h}$ em ágar MuellerHinton e as cepas de fungos a $35^{\circ} \mathrm{C}$ por $48 \mathrm{~h}$ em ágar Sabouraud Dextrose. Os testes foram realizados utilizando caldo MuellerHinton (MHB) para bactérias e caldo RPMI-1640 para fungos. Diluições sucessivas de 5,0 a 0,039 mg/mL dos extratos e frações foram preparadas em microplacas de 96 poços. Para isso foram utilizadas soluções estoque de $12,5 \mathrm{mg} / \mathrm{mL}$ em DMSO $1 \%$. Foram transferidos $80 \mu \mathrm{L}$ dessa solução para as microplacas, que já continham $100 \mu \mathrm{L}$ de meio de cultura. Para completar o volume final de $200 \mu \mathrm{L}$, foram adicionados $20 \mu \mathrm{L}$ de inóculo ( $10^{6}$ unidades formadoras de colônias (UFC/mL), de acordo 
com a escala turbidimétrica padrão de McFarland 0,5). As placas foram incubadas a $35^{\circ} \mathrm{C}$ por $24 \mathrm{~h}$ e $48 \mathrm{~h}$, respectivamente para bactérias e fungos. Os mesmos testes foram realizados simultaneamente com o controle positivo (MHB ou RPMI-1640 + microrganismo) e o controle negativo (MHB ou RPMI-1640 + extrato. Cloranfenicol (500 a 0,24 $\mu \mathrm{g} / \mathrm{mL})$ e anfotericina B (10 a $0,002 \mu \mathrm{g} / \mathrm{mL}$ ) foram usados como medicamentos de referência para bactérias e fungos, respectivamente. A CIM foi calculada como a menor diluição que apresentou completa inibição do crescimento do microrganismo testado. Todos os testes foram realizados em triplicatas.

\subsubsection{Avaliação quantitativa da atividade antimicrobiana}

De acordo com Eloff (2004), a atividade antimicrobiana total, que indica quantas vezes as substâncias biologicamente ativas, presentes em $1 \mathrm{~g}$ de planta, podem ser diluídas e ainda inibir o crescimento de microrganismos, foi calculada como a razão entre a quantidade de material extraído (em $\mathrm{mg}$ ) por $1 \mathrm{~g}$ de planta e a CIM.

Além da atividade total, o percentual de atividade e o índice de susceptibilidade microbiano (ISM) foram calculados de acordo com Bonjar (2004). O percentual de atividade demonstrou o potencial total antimicrobiano dos extratos. Este número representa a quantidade de cepas susceptíveis a uma determinada amostra e foi calculado como a razão entre número de cepas susceptíveis às amostras vegetais e o total de cepas microbianas testadas multiplicado por cem. Já o ISM foi usado para comparar a susceptibilidade relativa entre cepas microbianas e foi calculado como a razão entre o número de extratos efetivos contra cada cepa microbiana e o total de extratos vegetais testados multiplicado por cem. O valor de ISM varia de ' 0 ' (resistente a todos as amostras) até ' 100 ' (susceptíveis a todos as amostras). Os gráficos foram construídos por meio do programa Graph Prism 5.0.

\subsection{Atividade citotóxica frente a Artemia salina}

A citotoxicidade frente a Artemia salina foi avaliada utilizando-se a metodologia proposta por Rajabi et al. (2015). Larvas de A. salina (náuplios), obtidas a partir de ovos utilizados como ração para peixes ornamentais, foram colocadas para eclodir em aquário com água do mar artificial arejada durante 48h. As amostras foram solubilizadas em água do mar artificial, com a ajuda de um diluente (DMSO 1\%). Os testes foram realizados em tubos de ensaio com 10 náuplios, preenchidos com 4,5 $\mathrm{mL}$ de água do mar artificial e $0,5 \mathrm{~mL}$ do material a ser avaliado. As amostras foram testadas nas concentrações de 1000, 500, 250, 100 e $10 \mu \mathrm{g} / \mathrm{mL}$. Após 24 horas foram contados os indivíduos vivos nas diferentes concentrações. Timol e a água do mar artificial + DMSO $1 \%$ foram usados com controles positivo e negativo, respectivamente. Os testes foram realizados em triplicata. Os resultados foram expressos em $\mathrm{CL}_{50}$ (concentração necessária para matar 50\% dos náuplios) que foi calculado pelo programa estatístico Grafit 5.

\subsection{Atividade citotóxica frente a linhagens de células tumorais}

\subsubsection{Linhagem de células}

Duas linhagens de células tumorais humanas foram usadas: Jurkat (linhagem humana imortalizada dos linfócitos T) e HL60 (leucemia promielocítica humana). As células HL60 e Jurkat foram gentilmente cedidas pelo Dr. Gustavo AmaranteMendes (Universidade de São Paulo, Brasil). Todas as linhagens foram mantidas na fase logarítmica de crescimento em RPMI 1640 suplementado com $100 \mathrm{U} / \mathrm{mL}$ de penicilina e $100 \mathrm{mg} / \mathrm{mL}$ de estreptomicina, enriquecida com $2 \mathrm{mM}$ de L-glutamina e $10 \%$ de soro fetal bovino. Todas as culturas foram mantidas a $37{ }^{\circ} \mathrm{C} \mathrm{em}$ uma incubadora umidificada com $5 \% \mathrm{CO}_{2}$ e $95 \%$ de ar. Os meios foram alterados duas vezes por semana e eram regularmente examinados. 


\subsubsection{Avaliação do efeito citotóxico}

Linhagens de células tumorais (HL60 e Jurkat) foram inoculadas a $5 \times 10^{4}$ células por poço. As placas foram préincubadas por $24 \mathrm{~h}$ a $37^{\circ} \mathrm{C}$ para permitir a adaptação das células antes da adição das amostras. Uma solução preparada das amostras foi testada por $48 \mathrm{~h}$ em atmosfera de $\mathrm{CO} 2$ a $5 \%$ e $100 \%$ de umidade relativa. As amostras foram testadas na concentração de $20 \mu \mathrm{g} / \mathrm{mL}$. O veículo (DMSO a 0,05\%) e etoposídeo na concentração de $14 \mu \mathrm{M}$ foram utilizados como controle de crescimento (controle negativo) e controle positivo, respectivamente. A citotoxicidade (viabilidade celular) foi avaliada por meio do ensaio com 3-[4,5-dimetil-tiazol-2-il]-2,5-difeniltetrazólio (MTT) (Mosmann, 1983). Após 48h, os sobrenadantes foram descartados e $100 \mu \mathrm{L}$ de RPMI foram adicionados em todos os poços contendo as células cultivadas e em seguida $10 \mu \mathrm{L}$ de MTT $(5 \mathrm{mg} / \mathrm{mL})$ foi acrescentado a cada poço. A placa foi incubada a $37{ }^{\circ} \mathrm{C}$ em estufa com $5 \%$ de $\mathrm{CO}_{2}$ por 4h. Transcorrido esse período, a reação foi finalizada com a retirada do meio de cultura e adição de DMSO. A absorbância (Abs) foi lida a $570 \mathrm{~nm}$ e a viabilidade celular (\%) foi obtida pela seguinte fórmula: \% de células viáveis = Abs (Amostra)/Abs (DMSO 0,05\%) X 100. Sendo: Abs (Amostra): Absorbância mesurada após tratamento das células com as amostras; Abs (DMSO 0,05\%): Absorbância mesurada após tratamento das células com DMSO a 0,05\% (controle negativo). Para comparação, a citotoxicidade de etoposídeo $(14 \mu \mathrm{M})$ foi avaliado sob as mesmas condições experimentais. Todas as amostras foram testadas em três experimentos independentes (Monks et al., 1991).

\subsection{Análise estatística}

A análise estatística dos testes foi realizada pelo teste de ANOVA seguido de pós teste de Bonferroni, por meio do programa Graph Prism 5.0. Os valores de $\mathrm{p}<0,05$ foram considerados significativos. Os resultados foram expressos na forma de média \pm desvio padrão.

\section{Resultados}

As amostras MFAH e MFAM, apresentaram características organolépticas, cromatográficas e biológicas diferentes. O MFAH apresentou-se como um pó branco amorfo solúvel em água ou solução de acetonitrila:água (7:3, v/v). Em CCD foram observadas duas bandas com Rf de 0,75 e 0,13 sendo aquela com Rf maior revelada com o Reativo de Dragendorff, indicando a presença de alcaloides. Já MFAM apresentou-se como uma amostra escura pastosa, solúvel em metanol.

O fracionamento de MFAM em coluna aberta com Sephadex LH-20 forneceu 10 frações (MFAM1 a MFAM10) que foram submetidas a CCD. A cromatoplaca foi revelada com o reativo de Dragendorff e observou-se que os alcaloides concentraram-se nas frações de MFAM2 e MFAM3, sendo a maior concentração em MFAM2 (dados não mostrados). Esta fração foi submetida à CCD com fase móvel acidificada (diclorometano: metanol, 7:3 + HCl 0,5\%) para identificar quantos alcaloides possui. Após revelação com reativo específico verificou-se a presença de dois alcaloides com Rf 0,71 e 0,43.

$\mathrm{O}$ rendimento e a avaliação da atividade antimicrobiana de MFAH, MFAM e suas frações estão representados na Tabela 1. Foi possível observar que as frações ricas em alcaloides foram mais ativas aos microrganismos testados, com destaque para MFAM2, fração que obteve maior rendimento, que apresentou valores de CIM de 0,039 e 0,078 mg/mL, respectivamente para $S$. aureus, S. typhimurium, B. cereus, $P$. aeruginosa, $C$. neoformans e $S$. sonnei. Já MFAM3 apresentou atividade somente para B. cereus, P. aeruginosa e C. neoformans com valores de CIM de 0,039 e 0,078 mg/mL, respectivamente. 
Tabela 1. Rendimento (\%,p/p) e concentração inibitória mínima (CIM) das frações de alcaloides de Mitracarpus frigidus.

\begin{tabular}{|c|c|c|c|c|c|c|c|c|c|c|}
\hline \multirow[t]{2}{*}{ Amostras } & \multirow[t]{2}{*}{$\begin{array}{l}\text { Rendimento } \\
(\%, p / p)\end{array}$} & \multicolumn{7}{|c|}{$\begin{array}{l}\text { Atividade antibacteriana } \\
(\mathrm{CIM}-\mathrm{mg} / \mathrm{mL})^{\mathrm{a}}\end{array}$} & \multicolumn{2}{|c|}{$\begin{array}{l}\text { Atividade } \\
\text { antifúngica }(\mathrm{CIM} \\
-\mathrm{mgmL})^{\mathrm{a}}\end{array}$} \\
\hline & & $\mathrm{Sa}$ & Ec & St & Ss & $\mathrm{Kp}$ & $\mathrm{Bc}$ & $\mathrm{Pa}$ & $\mathrm{Ca}$ & $\mathrm{Cn}$ \\
\hline MFAH & $0,0023^{*}$ & 1,25 & 2,5 & 1,25 & 2,5 & 5,0 & 0,625 & 1,25 & 1,25 & 0,313 \\
\hline MFAM & $10,0 *$ & 0,156 & 0,625 & 0,625 & 0,313 & 1,25 & 0,156 & 0,156 & 0,625 & 0,078 \\
\hline MFAM1 & $0,77 * *$ & 0,625 & 1,25 & 1,25 & 0,313 & 2,5 & 0,313 & 0,313 & 0,625 & 0,156 \\
\hline MFAM2 & $49,54 * *$ & 0,039 & 0,313 & 0,039 & 0,078 & 0,313 & 0,039 & 0,039 & 0,313 & 0,039 \\
\hline MFAM3 & $26,92 * *$ & 1,25 & 2,5 & 0,313 & 0,313 & 0,313 & 0,039 & 0,078 & 0,625 & 0,078 \\
\hline MFAM4 & $3,85^{* *}$ & 5,0 & 5,0 & 2,5 & 0,625 & $>5,0$ & 0,156 & 0,313 & 2,5 & 1,25 \\
\hline MFAM5 & $2,31 * *$ & 2,5 & 5,0 & 5,0 & 2,5 & $>5,0$ & 1,25 & 1,25 & 5,0 & 0,156 \\
\hline MFAM6 & $4,30 * *$ & $>5,0$ & 5,0 & 5,0 & 1,25 & $>5,0$ & 5,0 & 5,0 & 5,0 & 1,25 \\
\hline MFAM7 & $1,00^{* *}$ & $>5,0$ & 5,0 & 5,0 & 2,5 & $>5,0$ & 5,0 & 5,0 & 5,0 & 1,25 \\
\hline MFAM8 & $3,85 * *$ & $>5,0$ & 5,0 & 5,0 & 5,0 & $>5,0$ & 2,5 & 5,0 & 5,0 & 1,25 \\
\hline MFAM9 & $3,62 * *$ & 5,0 & 5,0 & 2,5 & 5,0 & $>5,0$ & 2,5 & 5,0 & 5,0 & 1,25 \\
\hline MFAM10 & $0,46 * *$ & 1,25 & 5,0 & 2,5 & 5,0 & $>5,0$ & 5,0 & 5,0 & 5,0 & 1,25 \\
\hline Clor $^{b}$ & & 62,5 & 15,6 & 0,98 & 0,98 & 0,98 & 3,9 & 15,6 & & \\
\hline Anfot $^{\mathrm{b}}$ & & & & & & & & & 0,39 & 0,78 \\
\hline
\end{tabular}

* Rendimento em relação ao extrato bruto $(1,3 \mathrm{Kg})$.

** Rendimento relativo ao MFAM (1,3 g).

${ }^{a}$ Organismos testados: Sa, Staphylococcus aureus; Ec, Escherichia coli; St, Salmonella enterica sorovar typhimurium; Ss, Shigella sonnei; Kp, Klebsiella pneumoniae; Bc, Bacillus cereus; $\mathrm{Pa}$, Pseudomonas aeruginosa; $\mathrm{Ca}$, Candida albicans; Cn, Cryptococcus neoformans. ${ }^{\mathrm{b}}$ Controles positivos $(\mu \mathrm{g} / \mathrm{mL})$ : Clor - Cloranfenicol; Anfot - Anfotericina B.

Fonte: Autores (2021).

A atividade total $(\mathrm{mL} / \mathrm{g})$, o percentual de atividade e o ISM das amostras que apresentaram valores de CIM menores que $0,100 \mathrm{mg} / \mathrm{mL}$ estão apresentados na Tabela 2 e nas Figuras 1 e 2, respectivamente. MFAM apresentou melhor atividade total $(12,82 \mathrm{~mL} / \mathrm{g}$ para $C$. neoformans), seguido de MFAM2 (fração mais rica em alcaloides) com atividade total de 12,70 $\mathrm{mL} / \mathrm{g}$ para S. aureus, S. typhimurium, B. cereus, P. aeruginosa e C. neoformans. Além disso, MFAM2 apresentou melhor percentual de atividade em relação a todos os microrganismos testados (67\%). O cálculo do índice de susceptibilidade microbiana detectou que $C$. neoformans foi o microrganismo mais sensível às amostras testadas (25\%), seguido de B. cereus $(17 \%)$ e $P$. aeruginosa $(17 \%)$. 
Tabela 2. Atividade total (mL/g) das amostras testadas com valores de CIM inferiores a 0,100 mg/ mL.

\begin{tabular}{lll}
\hline Amostras & Microrganismos & Atividade total \\
\hline MFAM & Cryptococcus neoformans & 12,82 \\
MFAM2 & Staphylococcus aureus & 12,70 \\
& Salmonella typhimurium & 12,70 \\
& Shigella sonnei & 6,35 \\
& Bacillus cereus & 12,70 \\
& Pseudomonas aeruginosa & 12,70 \\
& Cryptococcus neoformans & 12,70 \\
& Bacillus cereus & 6,90 \\
& Pseudomonas aeruginosa & 3,45 \\
& Cryptococcus neoformans & 3,45 \\
\hline
\end{tabular}

Fonte: Autores (2021).

Figura 1. Percentual de atividade das amostras testadas em relação aos microrganismos mais susceptíveis (valores de CIM inferiores a $0,100 \mathrm{mg} / \mathrm{mL})$.

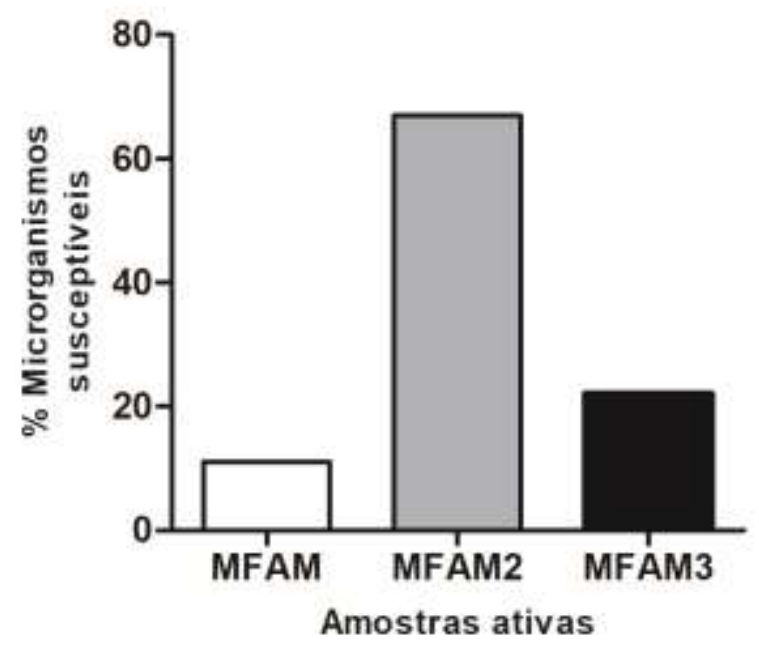

Fonte: Autores (2021). 
Figura 2. Índice de susceptibilidade microbiana (ISM) calculado a partir de valores CIM inferiores $0,100 \mathrm{mg} / \mathrm{mL}$ das amostras testadas.

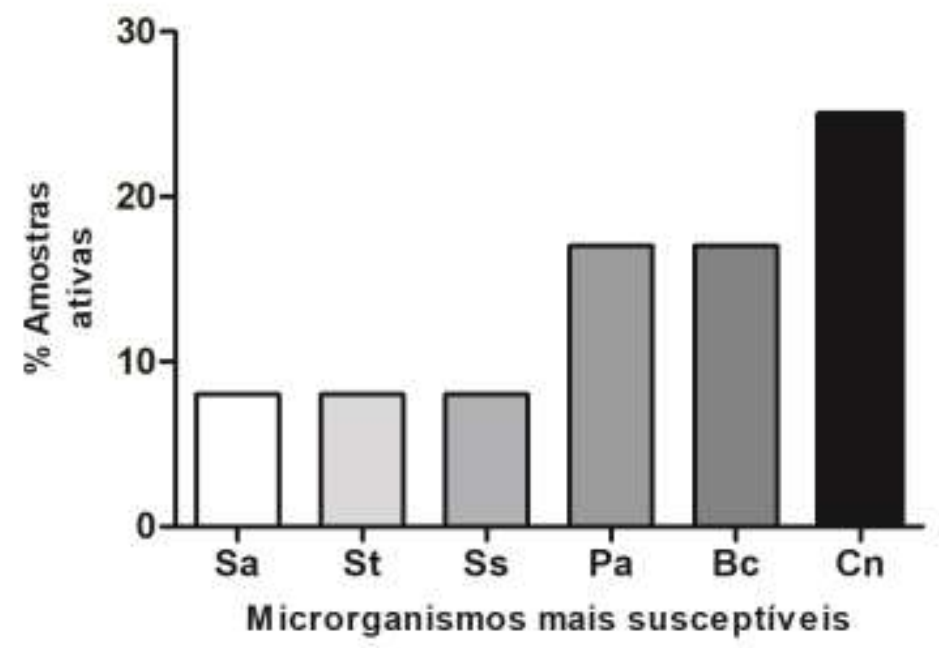

Microrganismos: Sa - Staphylococcus aureus; $\mathrm{St}$ - Salmonella enterica sorovar typhimurium; Ss - Shigella sonnei; $\mathrm{Bc}-$ Bacillus cereus; Pa Pseudomonas aeruginosa; $\mathrm{Cn}$ - Cryptococcus neoformans.

Fonte: Autores (2021).

As amostras testadas não apresentaram efeito citotóxico contra $A$. salina $\left(\mathrm{CL}_{50}>250,0 \mu \mathrm{g} / \mathrm{mL}\right)$. Já em relação as linhagens de células tumorais, MFAM2 e MFAM3 apresentaram moderada atividade citotóxica inibindo cerca de 50 e $35 \%$ da viabilidade celular de HL60 e Jurkart, respectivamente (Figura 3). Todas as amostras testadas apresentaram resultados significativos diferentes ao controle de crescimento (DMSO a 0,05\%) para ambas as células, com exceção de MFAH (p < $0,05)$.

Figura 3. Atividade antitumoral das amostras que deram positivo para presença de alcaloides frente a duas linhagens de células leucêmicas, HL60 e Jurkat.

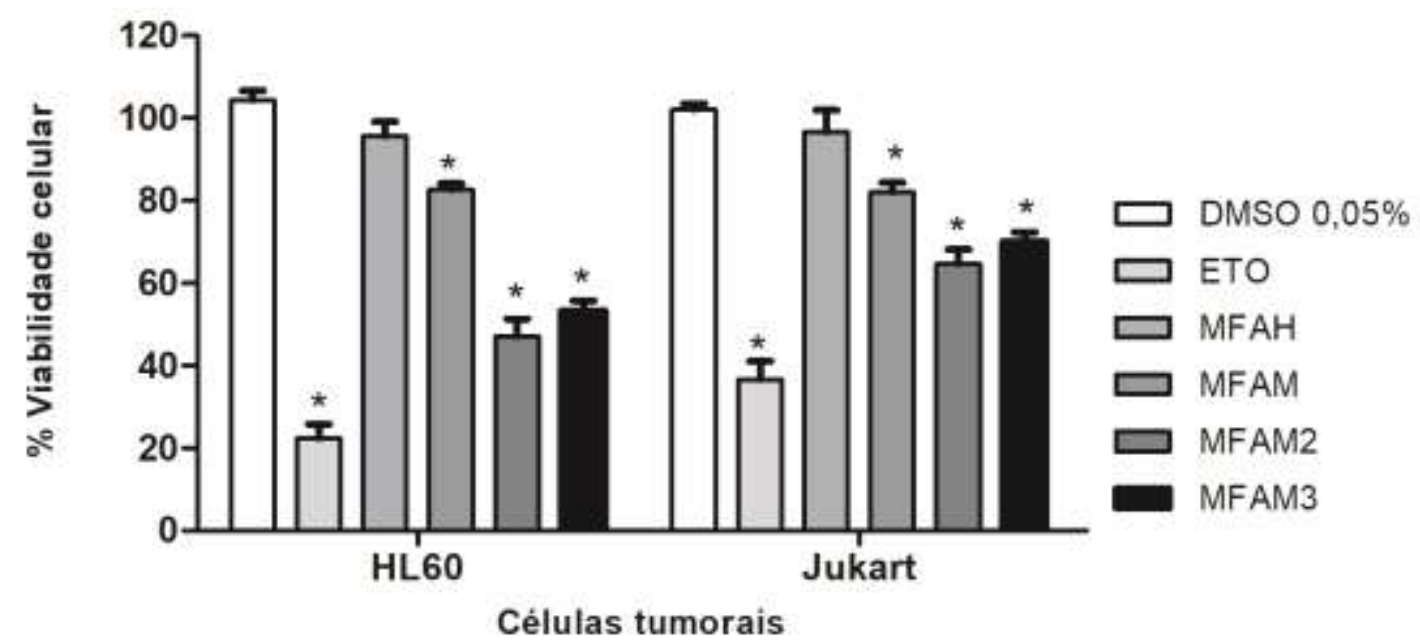

DMSO a 0,05\% - controle negativo; Etoposídeo (ETO) a $14 \mu \mathrm{M}$ controle positivo. *Estatisticamente diferente ao controle negativo (ANOVA seguido de Bonferroni, $\mathrm{p}<0,05$ ).

Fonte: Autores (2021). 


\section{Discussão}

A família Rubiaceae é rica em alcaloides indólicos e benzilisoquinolínicos para os quais já foram descritas inúmeras atividades biológicas (Martins \& Nunez, 2015; Rosales et al., 2020). Isso torna interessante o isolamento dos alcaloides de $M$. frigidus, pois existe uma grande possibilidade de serem encontrados alcaloides altamente bioativos. Dessa forma, os alcaloides foram extraídos das partes aéreas de $M$. frigidus e suas atividades antimicrobiana e citotóxica foram avaliadas.

MFAM apresentou moderada atividade antimicrobiana (Tabela 1) e por esse motivo foi fracionada em coluna aberta usando Sephadex como fase estacionária. As frações ricas em alcaloides, MFAM2 e MFAM3, foram as que apresentaram melhores resultados contra os microrganismos testados, com destaque MFAM2 que apresentou maior atividade total para $C$. neoformans, B. cereus e $P$. aeruginosa e, também maior percentual de atividade em relação a todos os microrganismos testados. Não se pode atribuir a atividade antimicrobiana dessas frações somente pela presença de alcaloides, mas como as demais frações não obtiveram resultados tão promissores, fortes indícios podem ser inferidos sobre uma possível atividade biológica desses compostos.

O ISM revelou que os microrganismos C. neoformans, B. cereus e P. aeruginosa foram os mais susceptíveis às amostras testadas, principalmente às fraçoes ricas em alcaloides. Estes resultados são de grande importância visto que esses microrganismos são causadores de diversas patologias. C. neoformans, nos últimos anos, têm recebido grande atenção dos pesquisadores por serem patógenos que afetam pacientes imunologicamente comprometidos, como aqueles com a síndrome de imunodeficiência adquirida (SIDA). Podem causar meningite criptocócica, que é a infecção mais comum de criptococose, e é geralmente crônica e fatal se não tratada a tempo (Stott et al., 2021). B. cereus é uma bactéria Gram-positiva contaminante de muitos alimentos podendo causar diarreia, dores abdominais, náuseas e vômitos devido à produção de enterotoxinas (Guven et al., 2005). P. aeruginosa é um bacilo Gram-negativo, podendo causar infecções localizadas, decorrentes de uma contaminação oportunista. Este microrganismo pode causar infecções urinárias e respiratórias, pneumonias, meningites, endocardites e diversos outros tipos de infecção. Além disso, P. aeruginosa, bem como outras espécies de Pseudomonas, apresentam grande importância para a indústria de alimentos, pois são microrganismos causadores de deterioração (Soberon et al., 2007).

As amostras foram avaliadas, inicialmente, quanto a sua citotoxicidade por meio do teste com A. salina, um ensaio de letalidade que tem sido usado em triagens rotineiras de toxicologia e que apresenta algumas vantagens por ser um ensaio rápido, econômico, simples e que possui forte correlação com a atividade antitumoral (Rajabi et al., 2015). Nenhuma amostra testada, extratos e frações apresentaram atividade citotóxica por este método. Após, foi realizado o ensaio de citotoxicidade em células tumorais pelo método de MTT, uma vez que é recomendado a realização de mais de um ensaio biológico para pesquisa de novos compostos antitumorais. O ensaio com as linhagens tumorais revelou uma redução da viabilidade celular tanto para HL60 quanto Jurkart quando comparado ao controle de crescimento $(\mathrm{p}<0,05)$. Esses resultados são promissores, uma vez que o câncer é uma patologia que possui altas taxas de mortalidade e incidência, levando a 17,5 milhões de casos em todo o mundo e 8,7 milhões de mortes apenas no ano de 2015 (Global Burden of Disease Cancer Collaboration, 2017). Outro fator importante é a busca de novos fármacos que possam atuar contra células tumorais e ter baixa toxicidade as células normais adjacentes, já que os agentes quimioterápicos convencionais causam graves efeitos adversos como cefaleia, sonolência, confusão mental, paralisia motora, dor nos membros inferiores, rigidez na nuca, convulsão, depressão, falência do miocárdio, hipotensão; fibrose pulmonar, tosse seca, dispneia, febre, perda de peso e calafrios, além de gerar resistência pelas células cancerígenas (Chen et al., 2014). Desta forma a busca de novas terapêuticas que diminuam esses efeitos adversos é incessante e, pesquisas mais aprofundadas com as frações ricas em alcaloides de Mitracarpus frigidus podem servir como estratégia futura de tratamento para o câncer. 


\section{Conclusão}

Mitracarpus frigidus se apresenta como uma fonte promissora de substâncias que possuem atividades biológicas como antimicrobiana e citotóxica. As frações ricas em alcaloides apresentaram significativa atividade para microrganismos oportunistas causadores de diversas doenças como meningite, além de apresentarem promissoras inibição de linhagens tumorais leucêmicas. Os resultados exibidos abrem perspectivas para o futuro isolamento, purificação e identificação das substâncias bioativas de M. frigidus que possam ser utilizadas no tratamento de infecções microbianas e do câncer.

\section{Agradecimentos}

Os autores agradecem a Fundação de Amparo à Pesquisa do estado de Minas Gerais (FAPEMIG) e a Universidade Federal de Juiz de Fora (UFJF) pelo suporte financeiro e ao Dr. Vinícius Antônio de Oliveira Dittrich do Departamento de Botânica da UFJF pela identificação botânica da espécie vegetal.

\section{Referências}

Bonjar, G. H. S. (2004). New approaches in screening for antibacterial in plants. Asian Journal of Plant Science, 3, 55-60.

Chen, C.; Hu, J., Zeng, P.; Pan, F., Yaseen, M., Xu, H. \& Lu, J. R. (2014). Molecular mechanisms of anticancer action and cell selectivity of short a-helical peptides. Biomaterials, 35, 1552-1561.

CLSI, Clinical and Laboratory Standards Institute (2010). Performance standards for antimicrobial susceptibility testing. Twenty first informational supplement. CLSI Document M100-S21.

CLSI, Clinical and Laboratory Standards Institute (2017). Performance standards for antimicrobial susceptibility testing. (4th ed.), CLSI Document M27.

Cordell, G. A., Quinn-Beattie, M. L. \& Farnsworth, N. R. (2001). The potential of alkaloids in drug discovery. Phytotherapy Research, 15, 183-205.

Eloff, J. N. (2004). Quantifying the bioactivity of plant extracts during screening and bioassay-guided fractionation. Phytomedicine, 11 , 370-371.

Fabri, R. L., Nogueira, M. S., Braga, F. G., Coimbra, E. S. \& SCIO, E. (2009). Mitracarpus frigidus aerial parts exhibited potent antimicrobial, antileishmaninal, and antioxidant effects. Bioresource Technology, 100, 428-433.

Gbaguidi, F., Accrombessi, G., Moudachirou, M. \& Quetin-Leclercq, J. (2005). HPLC quantification of two isomeric triterpenic acids isolated from Mitracarpus scaber and antimicrobial activity on Dermatophilus congolensis. Journal of Pharmaceutical and Biomedical Analysis, 39 , 990-995.

Global Burden of Disease Cancer Collaboration (2017). Global, regional, and national cancer incidence, mortality, years of life lost, years lived with disability, and disability adjusted life-years for 32 cancer groups, 1990 to 2015: A systematic analysis for the global burden of disease study. JAMA Oncology, 3(4), 524548 .

Guven, K., Mutlu, M. B. \& Avci, O. (2005). Incidence and characterization of Bacillus cereus in meat and meat products consumed in Turkey. Journal of Food Safety, 26, 30-40.

Martins, D. \& Nunez, C. V. (2015). Secondary metabolites from Rubiaceae species. Molecules, 20, 13422-13495.

Matos, F. J. A. (1997). Introdução à fitoquímica experimental. (2a ed.). Edições UFC. 141p.

Monks, A., Scudiero, D., Skehan, P., Shoemaker, R., Paull, K., Vistica, D., Hose, C., Langley, J., Cronise, P. \& Vaigro-Wolff, A. (1991). Feasibility of a highflux anticancer drug screen using a diverse panel of cultured human tumor cell lines. Journal of National Cancer Institute, 83 (11), $757-766$.

Mossman, T. (1983). Rapid colorimetric assay for cellular growth and survival: apllication for proliferation in citotoxicity. Journal of Immunological Methods, $65,55-63$.

Nok, A. J. (2002). Azaanthraquinone inhibits respiration and in vitro growth of long slender bloodstream forms of Trypanosoma congolense. Cell Biochemistry and Function, 20, 205-212.

Okunade, A. L., Clark, A. M., Hufford, C. D. \& Oguntimein, B. O. (1999). Azaanthraquinone; An antimicrobial alkaloid from Mitracarpus scaber. Planta Medica, 65, 447-448.

Pereira, Z. V., Carvalho-Okano, R. M. \& Garcia, F. C. P. (2006) Rubiaceae Juss. da Reserva Florestal Mata de Paraíso, Viçosa, MG, Brasil. Acta Botanica Brasilica, 20, 207-224.

Rajabi, S., Ramazani, A., Hamidi, M. \& Naji, T. (2015) Artemia salina as a model organism in toxicity assessment of nanoparticles. Journal of Pharmaceutical Sciences, 23(20), 1-6.

Rosales, P. F., Bordin, G. S., Gower, A. E.\& Moura, S. (2020) Indole alkaloids: 2012 until now, highlighting the new chemical structures and biological activities. Fitoterapia, 143, 104558. 
Research, Society and Development, v. 10, n. 11, e148101119541, 2021

(CC BY 4.0) | ISSN 2525-3409 | DOI: http://dx.doi.org/10.33448/rsd-v10i11.19541

Simões, C. M. O., Schenkel, E. P., Gosmann, G., Mello, J. C. P., Mentz, L. A. \& Petrovick, P. (2011). Farmacognosia: da planta ao medicamento. (6a ed.) Editora da UFSC, 765-791.

Soberon, J. R., Sgariglia, M. A. \& Sampietro, D. A. (2007). Antibacterial activity of plants extracts from northwestern Argentina. Journal of Applied Microbiology, 102(6), 1450-1461.

Stott, K. E., Loyse, A., Jarvis, J. N., Alufandika, M., Harrison, T. S., Mwandumba, H. C., Day, J. N., Lalloo, D. G., Bicanic, T., Perfect, J. R. \& Hope, W. (2021). Cryptococcal meningoencephalitis: time for action. The Lancet. Infectious Diseases, S1473-3099(20), 30771-30774. 